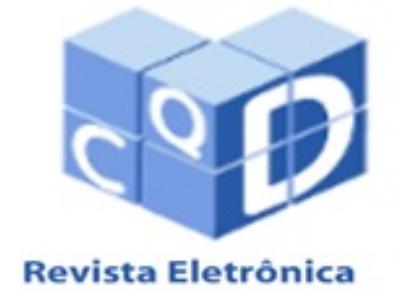

Paulista de Matemática

ISSN 2316-9664

Volume 9, jul. 2017

\section{Sequências de Cauchy de números fuzzy}

Cauchy Sequences of Fuzzy Numbers

\section{Silvio Antonio Bueno Salgado}

Universidade Federal de Alfenas- Campus Varginha silvio.salgado@unifalmg.edu.br

\section{Danilo Machado Pires}

Universidade Federal de

Alfenas - Campus Varginha danilo.pires@unifalmg.edu.br

\section{Leandro Ferreira}

Universidade Federal de Alfenas- Campus Varginha leandro.ferreira@unifalmg.edu.br

\section{Resumo}

O objetivo deste trabalho é apresentar uma discussão a respeito das sequências de Cauchy de números fuzzy, algumas de suas propriedades e exemplos.

Palavras-chave: Números Fuzzy. Sequências de Números Fuzzy. Sequências de Cauchy de Números Fuzzy.

\begin{abstract}
The objective of this work is to present a discussion about Cauchy sequences of fuzzy numbers, some of their properties and examples.

Keywords: Fuzzy Numbers. Fuzzy Numbers Sequences. Cauchy Sequences Fuzzy Numbers.
\end{abstract}




\section{Introdução}

A teoria dos conjuntos fuzzy foi introduzida em 1965, pelo matemático Lotfi A. Zadeh com o objetivo de dar um tratamento matemático a certos termos linguísticos subjetivos tais como: aproximadamente, em torno de, dentre outros. Dentre os conjuntos fuzzy, destacam-se os chamados números fuzzy, que são conjuntos fuzzy da reta real com certas propriedades especiais. Num certo sentido, os números fuzzy generalizam a idéia de número real.

Ao lidar com números fuzzy podemos pensar numa coleção desses objetos dispostos de maneira ordenada, ou seja, uma sequência de números fuzzy. Dada uma sequência definida sobre um conjunto $X$, para estudarmos questões como convergência, limitação e outras propriedades da sequência é necessário que o conjunto $X$ possua uma estrutura métrica. Matloka (1986) introduzui os conceitos de convergência para sequências de números fuzzy e estudou algumas de suas propriedades. Diversos autores vem estudando sequências de números fuzzy com diferentes propósitos. Nanda (1989) mostra que o espaço das sequências de números fuzzy que são limitadas e convergentes tem estrutura de espaço métrico completo. Nuray e Savas (1995) introduzem a idéia de sequências estatisticamente convergentes e estatisticamente Cauchy de números fuzzy e mostram a relação entre estes dois conceitos. Savas (2000) mostra que o conjunto das sequências de números fuzzy de diferenças limitadas é completo. Nuray (2008) introduz o conceito de I-convergência para sequências de números fuzzy. Recentemente, Altin e Çakan (2015) estenderam a noção de convergência estatística para sequências de números fuzzy.

Neste trabalho apresentamos uma discussão a respeito de uma classe especial de sequências de números fuzzy: as chamadas sequências de Cauchy de números fuzzy e também exibimos algumas propriedades notáveis e exemplos. Sequências de Cauchy de números fuzzy desempenham um papel fundamental em Análise Fuzzy. Tais sequências tem sido usadas extensivamente por diversos autores. Diamond e Kloeden (2000) utilizam sequências de Cauchy para mostrarem que o conjunto dos números fuzzy, munido da métrica de Hausdorff é um espaço métrico completo.

O trabalho está dividido da seguinte forma: na primeira seção apresentamos alguns conceitos fundamentais e notações indispensáveis para o estudo de sequências de números fuzzy. Na seção 2, definimos sequências de numeros fuzzy, convergência e estudamos algumas propriedades das mesmas. Já na seção 3, definimos sequências de Cauchy de números fuzzy, estudamos algumas de suas propriedades. Finalmente, apresentamos as referências que apoiaram a construção desse trabalho.

\section{Preliminares e notações}

Um número fuzzy é um subconjunto fuzzy de $\mathbb{R}$ cuja função de pertinência $u: \mathbb{R} \longrightarrow[0,1]$ atende as seguintes condições:

(i) $u$ é normal, isto é, existe $x_{0} \in \mathbb{R}$ tal que, $u\left(x_{0}\right)=1$;

(ii) $u$ é convexa, isto é, $u(t x+(1-t) y) \geq \min \{u(x), u(y)\}$, para todo $t \in[0,1]$;

(iii) $u$ é semicontínua superiormente (scs) em $\mathbb{R}$, isto é, dado $\varepsilon>0$, existe $\delta>0$ tal que, $\left|x-x_{0}\right|<\delta \Longrightarrow u(x)<u\left(x_{0}\right)+\varepsilon$;

(iv) $u$ tem suporte compacto, isto é, o conjunto $\mathrm{cl}\{x \in \mathbb{R} ; u(x)>0\}$ é compacto e $\mathrm{cl}(A)$ denota o fecho do conjunto $A$, com a topologia usual da reta. 
Denota-se por $\mathscr{F}(\mathbb{R})$ a coleção de todos os números fuzzy. Dado $u \in \mathscr{F}(\mathbb{R})$, o conjunto $\alpha$ - nível de $u$, denotado por $[u]^{\alpha}$ é defindo por

$$
[u]^{\alpha}=\{x \in \mathbb{R} ; u(x) \geq \alpha\}
$$

para todo $\alpha, 0<\alpha \leq 1$. Para cada $\alpha, 0<\alpha \leq 1$, o conjunto $[u]^{\alpha}$ é não vazio, fechado e limitado. Com isso, os $\alpha$ - níveis de $u$ serão denotados por

$$
[u]^{\alpha}=\left[u^{-}(\alpha), u^{+}(\alpha)\right] \text {. }
$$

Sejam $u, v, w \in \mathscr{F}(\mathbb{R})$ e $k \in \mathbb{R}$. Então as operações de adição, multiplicação por escalar e produto são definidos em $\mathscr{F}(\mathbb{R})$ por

(i) $u+v=w \Longleftrightarrow[w]^{\alpha}=[u]^{\alpha}+[v]^{\alpha}$ para todo $\alpha \in[0,1]$;

(ii) $[k . u]^{\alpha}=k \cdot[u]^{\alpha}$ para todo $\alpha \in[0,1]$;

(iii) $u . v=w \Longleftrightarrow[w]^{\alpha}=[u]^{\alpha} \cdot[v]^{\alpha}$ para todo $\alpha \in[0,1]$ em que

$$
\begin{aligned}
& w^{-}(\alpha)=\min \left\{u^{-}(\alpha) \cdot v^{-}(\alpha), u^{-}(\alpha) \cdot v^{+}(\alpha), u^{+}(\alpha) \cdot v^{-}(\alpha), u^{+}(\alpha) \cdot v^{+}(\alpha)\right\} \\
& w^{+}(\alpha)=\max \left\{u^{-}(\alpha) \cdot v^{-}(\alpha), u^{-}(\alpha) \cdot v^{+}(\alpha), u^{+}(\alpha) \cdot v^{-}(\alpha), u^{+}(\alpha) \cdot v^{+}(\alpha)\right\} .
\end{aligned}
$$

Seja $K$ a coleção de todos os intervalos fechados e limitados $A=\left[a_{1}, a_{2}\right]$ de $\mathbb{R}$. O conjunto $K$ tem estrutura de espaço vetorial induzida pelas operações

(i) $A+B=\{a+b ; a \in A, b \in B\}$;

(ii) $\lambda . A=\{\lambda . a ; a \in A\}$.

para quaisquer que sejam $A, B \in K$ e $\lambda \in \mathbb{R}$.

Para $A, B \in K$ o número real

$$
d(A, B)=\max \left\{\left|a_{1}-b_{1}\right|,\left|a_{2}-b_{2}\right|\right\}
$$

é chamado de distância de Hausdorff-Pompeiu dos intervalos $A$ e $B$.

Dados $u, v \in \mathscr{F}(\mathbb{R})$ a função

$$
D_{\infty}: \mathscr{F}(\mathbb{R}) \times \mathscr{F}(\mathbb{R}) \longrightarrow[0,+\infty[
$$

definida por

$$
D_{\infty}(u, v)=\sup _{0 \leq \alpha \leq 1} \max \left\{\left|u^{-}(\alpha)-v^{-}(\alpha)\right|,\left|u^{+}(\alpha)-v^{+}(\alpha)\right|\right\}
$$

é chamada de distância de Hausdorff entre os números fuzzy $u$ e $v$.

Proposição 1. (DIAMOND; KLOEDEN, 2000) Dados $u, v, w, z \in \mathscr{F}(\mathbb{R}) e k \in \mathbb{R}$, então:

(i) $O \operatorname{par}\left(\mathscr{F}(\mathbb{R}), D_{\infty}\right)$ é um espaço métrico;

(ii) $D_{\infty}(u+w, v+w)=D_{\infty}(u, v)$;

(iii) $D_{\infty}(k . u, k . v)=|k| D_{\infty}(u, v)$; 
(iv) $D_{\infty}(u+v, w+z) \leq D_{\infty}(u, w)+D_{\infty}(v, z)$.

Na literatura de Análise Fuzzy, é comum usarmos a distância de Hausdorff entre dois números fuzzy pois isso torna a estrutura desse espaço próxima da estrutura de um espaço de Banach. O conjunto dos números fuzzy não possui estrutura de espaço vetorial o que o impede de ser um espaço de Banach.

\section{Sequências de números fuzzy}

Todas as definições aqui apresentadas foram extraídas de Matloka (1986). Intuitivamente, uma sequência de números fuzzy é qualquer coleção de números fuzzy dispostos de maneira ordenada. Mais precisamente:

Uma sequência $\left\{u_{n}\right\}$ de números fuzzy é uma aplicação

$$
u_{n}: \mathbb{N} \longrightarrow \mathscr{F}(\mathbb{R})
$$

que a cada número natural $n$ associa um único número fuzzy $u_{n}$.

Uma subsequência de $\left\{u_{n}\right\}$ é uma restrição da aplicação $u_{n}: \mathbb{N} \longrightarrow \mathscr{F}(\mathbb{R})$ a um subconjunto infinito $\left\{n_{1}<n_{2}<\ldots<n_{k}<\ldots\right\}$ de $\mathbb{N}$.

A sequência $\left\{u_{n}\right\}$ de números fuzzy é dita limitada se o conjunto $\left\{u_{n} ; n \in \mathbb{N}\right\}$ de números fuzzy for um conjunto limitado, isto é, quando existem dois números fuzzy $v$ e $w$ tal que

$$
v \leq u_{n} \leq w,
$$

para qualquer que seja $n \in \mathbb{N}$.

A noção de convergência é fundamental no estudo de sequências de números fuzzy.

A sequência $\left\{u_{n}\right\}$ de números fuzzy converge para o número fuzzy $u_{0}$ se para cada $\varepsilon>0$, existir um número inteiro positivo $n_{0}$ tal que

$$
D_{\infty}\left(u_{n}, u_{0}\right)<\varepsilon
$$

para todo $n>n_{0}$. Caso a sequência $\left\{u_{n}\right\}$ de números fuzzy convirja para o número fuzzy $u_{0}$, denotaremos esse fato por

$$
u_{n} \longrightarrow u_{0}, \text { ou, } n \longrightarrow \infty, \text { ou, } \lim _{n \rightarrow \infty} u_{n}=u_{0} .
$$

Caso a sequência $u_{n}$ de números fuzzy não convirja, então diremos que a sequencia é divergente.

Proposição 2. [Unicidade do Limite] Uma sequência de números fuzzy não pode convergir para dois limites diferentes.

Demonstração. Seja $\left\{u_{n}\right\}$ uma sequência de números fuzzy e sejam $u_{0}, v_{0} \in \mathscr{F}(\mathbb{R})$ tais que

$$
u_{n} \longrightarrow u_{0} \quad \mathrm{e} \quad v_{n} \longrightarrow v_{0} \text { quando, } n \longrightarrow \infty .
$$

Então, pela definição de convergência, dado $\varepsilon>0$, existe $n_{0} \in \mathbb{N}$ tal que 


$$
D_{\infty}\left(u_{n}, u_{0}\right)<\frac{\varepsilon}{2} \quad \text { para, } \quad n>n_{0}
$$

Existe também $n_{1} \in \mathbb{N}$ tal que

$$
D_{\infty}\left(u_{n}, v_{0}\right)<\frac{\varepsilon}{2} \quad \text { para, } \quad n>n_{1} .
$$

Escolhendo $n=\max \left\{n_{0}, n_{1}\right\}$, segue que

$$
D_{\infty}\left(u_{0}, v_{0}\right) \leq D_{\infty}\left(u_{n}, u_{0}\right)+D_{\infty}\left(u_{n}, v_{0}\right)<\varepsilon,
$$

e, com isso,

$$
0 \leq D_{\infty}\left(u_{0}, v_{0}\right)<\varepsilon
$$

$\mathrm{e}$, portanto, $u_{0}=v_{0}$.

A próxima proposição nos conta que se uma sequência de números fuzzy converge, então a sequência de seus $\alpha$ - níveis também converge.

Proposição 3. Se a sequência $\left\{u_{n}\right\}$ de números fuzzy converge para o número fuzzy $u_{0}$, então a sequência $\left\{\left[u_{n}\right]^{\alpha}\right\}$ converge para $\left[u_{0}\right]^{\alpha}$ para todo, $\alpha \in[0,1]$.

Demonstração. Como $u_{n} \longrightarrow u_{0}$ quando $n \longrightarrow+\infty$, então dado $\varepsilon>0$, existe um inteiro positivo $n_{0}$ tal que

$$
D_{\infty}\left(u_{n}, u_{0}\right)<\varepsilon, n>n_{0}
$$

Por outro lado,

$$
D_{\infty}\left(u_{n}, u_{0}\right)=\sup _{0 \leq \alpha \leq 1} d\left(\left[u_{n}\right]^{\alpha},\left[u_{0}\right]^{\alpha}\right)<\varepsilon
$$

para todo $\alpha \in[0,1]$ e $n>n_{0}$, o que prova o resultado.

Proposição 4. (MATLOKA, 1986) Toda sequência convergente de números fuzzy é limitada.

\section{Sequências de Cauchy de números fuzzy}

Uma sequência $\left\{u_{n}\right\}$ de números fuzzy é de Cauchy se, para todo $\varepsilon>0$, existir um número inteiro positivo $n_{0}$ tal que

$$
D_{\infty}\left(u_{m}, u_{n}\right)<\varepsilon
$$

para $m, n>n_{0}$.

O conceito de sequência de Cauchy é um conceito métrico, ou seja, sua definição depende de uma métrica adotada num certo espaço. Note que a diferença dessa definição para a definição de sequência convergente é que trocamos o número fuzzy fixo $u_{0}$ por termos da própria sequência de números fuzzy. A seguir, apresentaremos algumas propriedades notáveis das sequências de Cauchy de números fuzzy.

SALGADO, S. A. B.; PIRES, D. M.; FERREIRA, L. Sequências de Cauchy de números Fuzzy. C.Q.D.- Revista Eletrônica Paulista de Matemática, Bauru, v. 
Proposição 5. Toda sequência convergente de números fuzzy é de Cauchy.

Demonstração. Seja $\left\{u_{n}\right\}$ uma sequência convergente de números fuzzy. Então, dado $\varepsilon>0$, existe um inteiro positivo $n_{0}$ tal que

$$
D_{\infty}\left(u_{n}, u_{0}\right)<\frac{\varepsilon}{2}
$$

para $n>n_{0}$. Para $m, n>n_{0}$, temos

$$
D_{\infty}\left(u_{m}, u_{n}\right) \leq D_{\infty}\left(u_{m}, u_{0}\right)+D_{\infty}\left(u_{n}, u_{0}\right)<\frac{\varepsilon}{2}+\frac{\varepsilon}{2}=\varepsilon
$$

o que mostra que $\left\{u_{n}\right\}$ é uma sequência de Cauchy.

Proposição 6. Toda sequência de Cauchy de números fuzzy é limitada.

Demonstração. Seja $\left\{u_{n}\right\}$ uma sequência de Cauchy de números fuzzy. Então, dado $\varepsilon>0$, existe um inteiro positivo $n_{0}$ tal que

$$
D_{\infty}\left(u_{m}, u_{n}\right)<\varepsilon
$$

para $m, n,>n_{0}$. Em particular, para $\varepsilon=1, D_{\infty}\left(u_{m}, u_{n}\right)<1$, para $m, n>n_{0}$. Logo o conjunto $X=\left\{u_{n_{0}+1}, u_{n_{0}+2}, \ldots\right\}$ é limitado. Como o conjunto $Y=\left\{u_{1}, \ldots, u_{n_{0}}\right\}$ também é limitado, segue que $X \cup Y$ também é limitado. Portanto, a sequência de Cauchy $\left\{u_{n}\right\}$ é limitada.

Proposição 7. Uma sequência de Cauchy de números fuzzy que possui uma subsequência convergente é convergente (e tem o mesmo limite que a subsequência).

Demonstração. Sejam $\left\{u_{n}\right\}$ uma sequência de Cauchy de números fuzzy e $\left\{u_{n_{k}}\right\}$ uma subsequência de $\left\{u_{n}\right\}$ que converge para $u_{0} \in \mathscr{F}(\mathbb{R})$. Dado $\varepsilon>0$, existe um inteiro positivo $n_{1}$ tal que

$$
D_{\infty}\left(u_{n_{k}}, u_{0}\right)<\frac{\varepsilon}{2}
$$

para $n_{k}>n_{1}$. Como a sequência $\left\{u_{n}\right\}$ é de Cauchy, então dado $\varepsilon>0$, existe um inteiro positivo $n_{2}$ tal que

$$
D_{\infty}\left(u_{m}, u_{n}\right)<\frac{\varepsilon}{2}
$$

para $m, n>n_{2}$. Seja $n_{0}=\max \left\{n_{1}, n_{2}\right\}$. Para qualquer que seja $n>n_{0}$, existe um inteiro positivo $n_{k}$ tal que $n_{k}>n$. Como, em particular, $u_{n_{k}}$ é termo da sequência $\left\{u_{n}\right\}$ segue que

$$
D_{\infty}\left(u_{n}, u_{0}\right) \leq D_{\infty}\left(u_{n}, u_{n_{k}}\right)+D_{\infty}\left(u_{n_{k}}, u_{0}\right)<\varepsilon,
$$

o que mostra que a sequência de Cauchy $\left\{u_{n}\right\}$ converge para $u_{0}$. 
Exemplo 8. A sequência de números fuzzy, extraída de Matloka (1986)

$$
u_{n}(x)=\left\{\begin{array}{l}
\frac{n}{2 n-1} x \text { se } 0 \leq x<\frac{2 n-1}{n} \\
1 \text { se } \frac{2 n-1}{n} \leq x \leq \frac{2 n+1}{n} \\
-\frac{n}{2 n-1}(x-4) \text { se } \frac{2 n+1}{n}<x \leq 4 \\
0 \text { caso contrário }
\end{array}\right.
$$

converge para o número fuzzy

$$
u_{0}(x)=\left\{\begin{array}{l}
-\frac{1}{2} x \text { se, } 0 \leq x<2 \\
-\frac{1}{2}(x-4) \text { se, } 2 \leq x \leq 4 \\
0 \text { caso contrário }
\end{array}\right.
$$

e, portanto, é uma sequência de Cauchy.

A Figura 1 ilustra geometricamente alguns termos da sequência $u_{n}$ de números fuzzy do exemplo anterior.

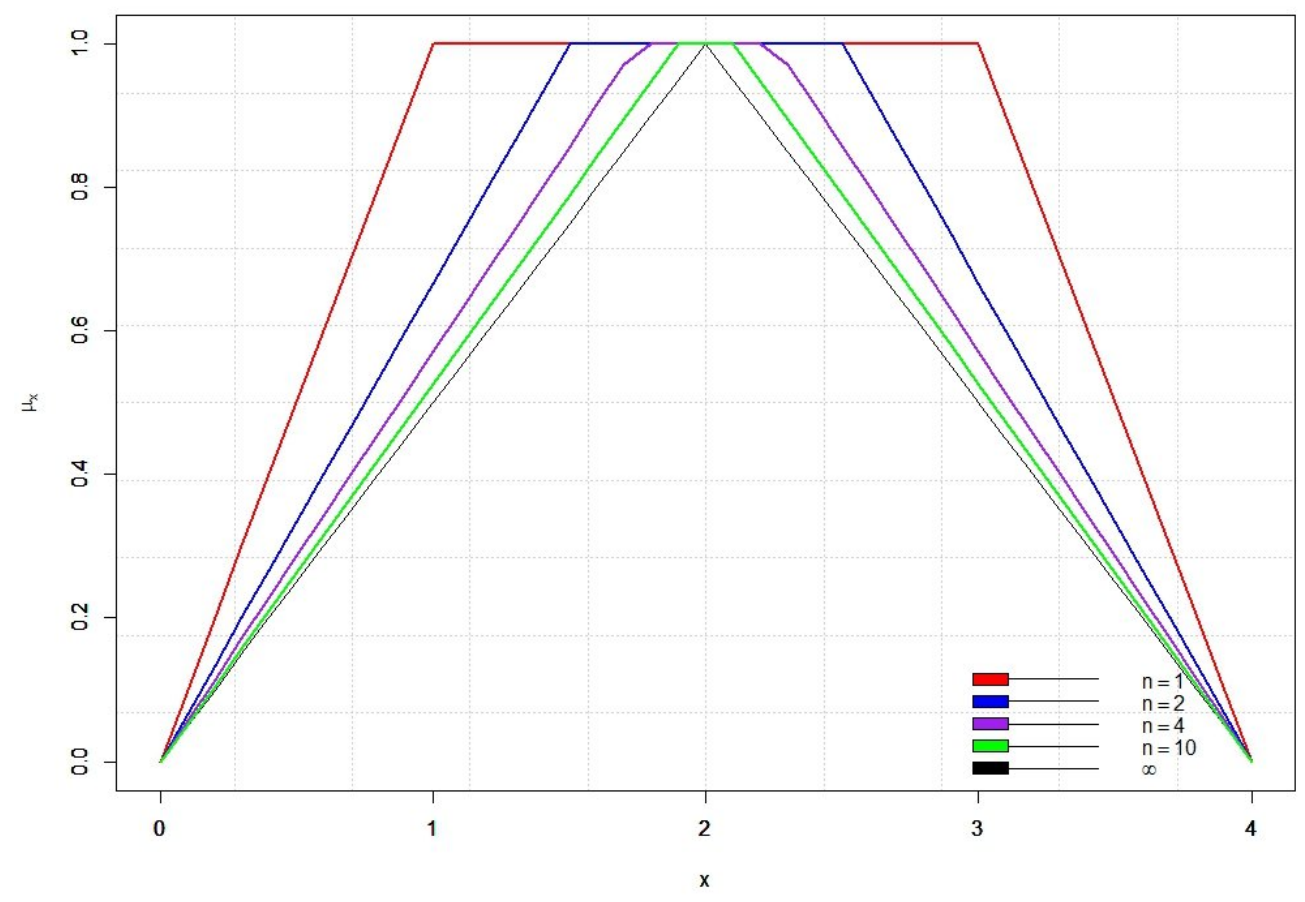




\section{Referências bibliográficas}

[1] ALTIN, Y.; ÇAKAN, U. Some classes of statistically convergent sequences of fuzzy numbers generated by a mudulus function. Iranian Journal of Fuzzy Systems, v. 12, n. 3, p. 47-55, 2015.

[2] DIAMOND, P.; KLOEDEN, P. Metric topology of fuzzy numbers and fuzzy analysis. In: DUBOIS, D.; PRADE, H. M. Fundamentals of fuzzy sets. Boston; Dordrecht: Kluwer Academic Publishers, 2000. p. 583-641. (The handbook of fuzzy sets series, v. 7).

[3] MATLOKA, M. Sequences of fuzzy numbers. Busefal, v. 28, p. 28-37, 1986.

[4] NANDA, S. On sequence of fuzzy numbers. Fuzzy Sets and Systems, v. 33, n. 1, p. 123-126, 1989.

[5] NURAY, F. I-convergence of sequences of fuzzy numbers. New Mathematics and Natural Computation, v. 4, n. 2, p. 231-236, 2008.

[6] NURAY, F.; SAVAS, E. Statistical convergence of sequences of fuzzy numbers. Math. Slovaca, v. 45, n. 3, p. 269-273, 1995.

[7] SAVAS, E. A note on sequence of fuzzy numbers. Information Sciences, v. 124, p. 297-300, 2000. 